\title{
The Place of Mental Health Rhetoric Research (MHRR) in Rhetoric of Health \& Medicine and Beyond
}

\author{
Cathryn Molloy, Drew Holladay \\ and Lisa Melonçon
}

In the inaugural issue of $R H M$, the editors invited J. Fred Reynolds (2018) to compose a "A Short History of Mental Health Rhetoric Research (MHRR)" in which he compellingly documented the "significant body of work applying the tools and terms of rhetoric to the world of mental health" that emerged in the 1980s and continued through the decades that followed, if in fits and starts (p. 1). As Reynolds documented, in the past, rhetoricians have studied issues of mental health from a variety of (inter)disciplinary angles: technical/professional writing vantages (Reynolds \& Mair, 2013; Berkenkotter, 2008; Holladay, 2017); critiques of the linguistic entanglements of the professionals who seek to treat mental health (McCarthy \& Gerring, 1994; Berkenkotter \& Ravotas, 1997); examinations of how publics encounter and make sense of mental difference (Leweicki-Wilson, 2003; Segal, 2008; Emmons, 2010; J. Johnson, 2010; Price, 2011; Rothfelder \& Thornton, 2017); and through studies of "patients" discursive behaviors (Prendergast, 2001; Molloy, 2015; Uthappa, 2017).

Reynolds' history raises important questions on how the issues and challenges unique to MHRR create space for the field to set a specific agenda for its development-to make explicit the major epistemological

(c) 2020 University of Florida Press 


\section{Editors' Introduction}

assumptions, the key questions, and the various vantage points that will undergird the future of this important area of inquiry. Reynolds' remarks, thus, helped to sketch out the history of MHRR as well as to establish it as an important research area for the future of $R H M$. While a number of fields examine issues of mental health from a humanistic perspective, rhetorical research on mental health distinguishes itself through a focus on discursive and symbolic communication-especially acts of persuasion and identification. Rhetorical approaches are not limited to textual analysis, however, and also account for factors such as social conditions, identity, embodiment, power relations, location, materiality, and circulation. MHRR, thus, attends to the rhetorics of neuroscience, medicine, and psychiatry in connection with their cultural warrants; places judgments of in/sanity in rhetorical-historical context; follows mental health categories and diagnoses through clinical, professional, and personal settings; considers representations of mental health in medical and professional documents as well as popular media; and connects rhetorical appeals to strategies of activism and advocacy.

This special issue, in many ways, builds and expands on that work by bringing together a diversity of rhetorical perspectives on mental healthcompositions that will undoubtedly inspire more work in this crucial area of study. Rather than introducing a new area that RHM scholars might start to consider, though, this special issue continues important work that has already been taking place in the journal and solidifies a commitment from the journal's editors to focus on this extremely important topic going forward. In their introduction to the inaugural issue, editors Lisa Melonçon and J. Blake Scott (2018) point out that RHM is a "dwelling place" for work in the area (p. ix). Emphasizing that "the capaciousness of rhetoric is only matched by the capaciousness of 'health' and 'medicine"' (iv), they are also clear that the journal's purpose is to establish a flexible set of boundaries for the field. In the same way, we set out to produce a special issue that could begin to clarify "flexible boundaries" for MHRR.

Arguably, few research topics in the rhetoric of health and medicine are as rich and incendiary as are mental health-related inquiries. Shrouded in etiological mystery - considered dubious social creations by some and serious brain diseases by others-mental health conditions have much in common with the complicated rhetorical lives of other contested diagnoses and 
chronic conditions. ${ }^{1}$ Symptoms of mental health "problems" are prescribed targeted interventions in the form of medications and therapies on the one end of the spectrum and are celebrated as rich sources of neural diversity on the other, making them quite similar to discourses surrounding disabilities writ large.

That is, mental health-related diagnoses name largely abstract behavioral symptoms and traits that some may call "ill," but others may call these same features "affordances." With so much contestation over what, exactly, constitutes mental "illness" and mental "wellness," rhetorical theories are especially appropriate lenses through which to examine this complex milieu. In the introduction to RHM's first special issue-on public healthco-editors Jennifer Malkowski and Lisa Melonçon (2019) astutely note that "a rhetorical orientation toward the study, practices, and communication of public health emphasizes how language helps to create, organize, challenge, and fragment public health realities" (p. iv). Building on this important work, this second special issue-on mental health rhetoric research (MHRR) - emphasizes the roles language and persuasion play in creating, reproducing, challenging, and altering mental health realities.

\section{Summary of Special Issue}

As J. Blake Scott (2017) helpfully explains, works published in this journal "draw on multiple scholarly traditions to develop new interdisciplinary theories, methodologies, and insights that can impact our understanding of health, illness, healing, and wellness" (n.p.) In keeping with the scope of $R H M$, the essays in this special issue draw on the scholarly traditions of rhetoric and writing; technical communication; and disability studies. Drawing on such interdisciplinary theories, contributors to this special issue make use of a diverse set of methods and methodologies, including empirical and observational work, historiography, close textual analysis, and theoretical interpretive work. These orientations simultaneously advance $R H M$ 's commitment to methodological innovation and emphasize the importance of such features in MHHR.

In the lead article in this special issue, Fernando Sánchez uses rhetorical context flowcharts and network pictures from his case study of a mental

${ }^{1}$ The 2020 winter issue of $R H M$ will be a special on chronic conditions. 


\section{Editors' Introduction}

health call center practitioner to coin and to begin theorizing what he is calling "distributed and mediated ethos." Sánchez's important new term describes how organizations that must offer essential health services at a distance rely on various resources to expand and control their identities across disparate locations and via various nodes. Among the constituent elements of a distributed and mediated ethos that Sánchez identifies are: projecting the impression of being "always there"; relying on dexterity across several human and nonhuman actors; and necessitating targeted tasks from branches that extend ethos farther from the organization. As RHM scholars grapple with how rhetorical lenses might clarify and enhance healthcare that is characterized by diffuse and shifting ontologies, we see potential in Sánchez's contribution to energize future work.

Next, readers will find Pam Takayoshi's study of 19th century women's firsthand accounts of their experiences in psychiatric hospitals. Using 23 women's memoirs, most of which are notably only accessible in archives, Takayoshi shows how the women asserted themselves as astute users of rhetoric; she argues, in fact, that these memoirs might even constitute the first extant patients' rights movement in the U.S. As Takayoshi explains, these women took back their power through rhetoric. Readers will note the author's novel use of data coding to create a pastiche of these recovered memoirists' voices. Impressive for its creative methodological approach as well as its findings, this essay would work quite well as a reading in a variety of courses in rhetoric.

Readers will also find Lenny Grant's historic tracing of the origins of post-traumatic stress disorder (PTSD) from its roots in "Post-Vietnam Syndrome" (PVS). Relying on constitutive rhetorical analyses of diverse archival materials, Grant argues that the PTSD diagnostic category grew directly out of activist efforts to reduce stigma and to increase empathy for Vietnam war veterans by slating them as psychologically injured survivors of war in direct opposition to doxa that painted them as murderers or junkies or both. This essay suggests that all future veterans of modern conflicts benefited from the creation of PTSD as a diagnostic category (and, hence, from psychiatrists' and others' activism) since PTSD carries the capacity to render a more favorable ethos for those who've experienced wartime combat.

Extending Lucille McCarthy and Joan Gerring's (1994) work on the $D S M$ as a "charter document" for the discipline of psychiatry, the next research article is Patty Kelly's analysis of the DSM-III's chief style attributes, which she convincingly argues constitute a "handbook of usage." Kelly's essay 


\section{Molloy et al.}

shows that the forms of textual standardizations of the profession's diagnostic manual that happened in the DSM III were pivotal in moving the field of psychiatry from psychoanaly tically inflected language to "neo-Kraepelinian" language. As such, says Kelly, the DSM-III textually solidified the principle professional attributes of psychiatry. Kelly's work will certainly resonate with other RHM scholarship that seeks to link textual practices in technical documents with epistemological allegiances of health and medical fields.

We are pleased to also feature an online dialogue, available at https:// stars.library.ucf.edu/rhm/vol3/iss2/, that enters into discussions of academic ableism. Lead author, Ann Green, along with Rebecca Carrasco, Lucía Dura, Patrick Harris, Leah Heilig, Alyssa Hilary, Bailey Kirby, Jay McClintick, and Emily Pfender bravely share their experiences of diagnosis and of their decisions on whether to disclose their diagnoses to students and colleagues. In this rich discussion, the authors meditate on how their "symptoms" are sometimes resources and other times hinderances on the job; they contribute to work that shows how academic culture tries to exclude those with mental health differences, yet these voices come together in this dialogue to combat some of the silence that leads to such oppression.

While the potential threads for studying MHRR feel limitless, the work collected in this special issue brought to the forefront a cohesion in three common themes that we see as the major areas of emphasis in MHRRstigma, institutional practices, and personal advocacy:

- The consistent focus on how stigma variously produces and reproduces mental health realities;

- The prevalence of institutional practices in the creation, deployment, and revision of mental health rhetorics; and

- The role of personal advocacy in intervening in systems of oppression related to mental health.

Thinking of stigma, institutional practices, and acts of personal advocacy as constituting a flexible organizing principle for MHRR work helps to establish a unifying rhetorical aim for this body of scholarship. Simultaneously, thinking in terms of consistent themes still leaves space for the possibility of multiple objects of rhetorical study and methodological approaches. As Scott and Melonçon (2018) maintain in their introduction to volume 1, issue 3-4, "a rhetorically anchored yet multidisciplinary journal like $R H M$... must embody and reinforce the key characteristics of 


\section{Editors' Introduction}

RHM scholarship but also remain open to new versions of it and expanded audiences and uses for it" (iv). Stigma, to be sure, is an ongoing problem; efforts to alleviate it have been robust in the areas of effort and energy, yet not as successful as authors of these efforts might have hoped (Hinshaw, 2006). With a focus on stigma, on institutional practices, and on personal advocacy, the works in this special issue continue RHM's firmly established dedication to examining the rhetorical dimensions of mental health. This special issue promises to contribute to interdisciplinary conversations on efforts to unpack and end stigma, on discourses surrounding institutional practices related to mental illness and wellness, and on the possibilities and limitations of forms of personal advocacy related to mental health.

As new work emerges from the energy generated by this special issue, we hope to see more work that takes up such vital issues as mental health stigma and its relation to health/help-seeking behaviors related to mental well-being. That is, recent interdisciplinary research on mental health and stigma suggests that stigma inhibits health/help-seeking behaviors (see, for example, Clement et al., 2015; Schnyder, Panczak, Groth, \& SchultzeLutter, 2017). Researchers might also examine other forms of stigma (i.e., race, gender, and social class-based stigmas) and how these other forms of oppression cross-pollinate with mental health-related stigmas to create the conditions of overdiagnosis, misdiagnosis, and poor quality of care. Another research area that would be most welcome in MHRR is rhetorical perspectives from in-the-trenches mental healthcare providers. As researchers continue to clarify the role MHRR plays in larger conversations to do with mental disabilities and in disability studies writ large, more work that explicitly examines and illuminates neurodiversities would be very beneficial to this area of research.

In sum, unlike some special issues that might be sort of "one off" engagements with interesting fringe topics, this is no "flash in the pan." This special issue is, of course, imperfect since one issue cannot account for all of the work being done in the area. Instead, it offers notable examples of the range of topics and approaches that scholars are considering in this vibrant arm of RHM work. Readers can expect to see RHM continue to offer researchers in MHRR space to share their important work.

\section{References}

Berkenkotter, Carol. (2008). Patient tales: Case histories and the uses of narrative in psychiatry. University of South Carolina Press. 


\section{Molloy et al.}

Berkenkotter, Carol \& Ravotas, Doris. (1997). Genre as tool in the transmission of practice over time and across disciplinary boundaries. Mind, Culture, and Activity 4(4), 256-274.

Clement, S., Schauman, O., Graham, T., Maggioni, F., Evans-Lacko, S., Bezborodovs, N., Morgan, C, Rusch, N., Brown, J., Thornicroft, G. (2015). What is the impact of mental health-related stigma on help-seeking? A systematic review of quantitative and qualitative studies. Psychological Medicine, 45(1), 11-27.

Emmons, Kimberly. (2010). Black dogs and blue words: Depression and gender in the age of self-care. Rutgers University Press.

Hinshaw, Stephen P. (2006). The mark of shame: Stigma of mental illness and an agenda for change. Oxford University Press.

Holladay, Drew. (2017). Classified conversations: Psychiatry and tactical technical communication in online spaces. Technical Communication Quarterly, 26(1), $8-24$.

Johnson, Jennell. (2010). The skeleton on the couch: The Eagleton affair, rhetorical disability, and the stigma of mental illness. Rhetoric Society Quarterly, 40(5), 459-478.

Lewiecki-Wilson, Cynthia. (2003). Rethinking rhetoric through mental disabilities. Rhetoric Review, 22(2), 156-167. https://www.jstor.org/stable /3093036

Malkowski, Jennifer and Melonçon, Lisa. (2019). The rhetoric of public health for RHM scholarship and beyond. Rhetoric of Health and Medicine, 2(2), iii-xiv.

McCarthy, Lucille P., \& Gerring, Joan Page. (1994). Revising psychiatry's charter document: DSM-IV. Written Communication, 11(2), 147-192.

Melonçon, Lisa \& Scott, J. Blake. (2018). Manifesting a scholarly dwelling place in RHM. Rhetoric of Health $\mathcal{\sigma}^{\circ}$ Medicine, 1(1-2), x.

Molloy, Cathryn. (2015). Recuperative ethos and agile epistemologies: Toward a vernacular engagement with mental illness ontologies. Rhetoric Society Quarterly, 45(2), 138-163.

Prendergast, Catherine. (2001). On the rhetoric of mental disability. In J. C. Wilson (Ed.), Embodied rhetoric: Disability in language and culture (pp. 47-60). Southern Illinois University Press.

Price, Margaret. (2011). Mad at school: Rhetorics of mental disability and academic life. University of Michigan Press.

Reynolds, J. Fred. (2018). A short history of mental health rhetoric research (MHRR). Rhetoric of Health $\xi^{\circ}$ Medicine, 1(1-2), 1-18.

Reynolds, John Frederick \& Mair, David C. (2013). Writing and reading mental health records: Issues and analysis in professional writing and scientific rhetoric. Routledge. https://www.taylorfrancis.com/books/9781136687488

Rothfelder, Katy \& Thornton, Davi Johnson. (2017). Man interrupted: Mental illness narrative as a rhetoric of proximity. Rhetoric Society Quarterly, 47(4), 359-382. 


\section{Editors' Introduction}

Schnyder, N., Panczak, R., Groth, N., \& Schultze-Lutter, F. (2017). "Association between mental health-related stigma and active help-seeking: Systematic review and meta-analysis": Correction. The British Journal of Psychiatry, 211(3), 184-184.

Scott, J. Blake (2017). Rhetoric of Health and Medicine Journal: The " $R$ " in Rhetoric of Health and Medicine. Dir. Scott, J. Blake. Perf. Scott, J. Blake. YouTube.

Segal, Judy Z. (2008). Health and the rhetoric of medicine. Southern Illinois University Press. http://ebookcentral.proquest.com/lib/jmu/detail.action?docID $=1354472$

Uthappa, N. Renuka. (2017). Moving closer: Speakers with mental disabilities, deep disclosure, and agency through vulnerability. Rhetoric Review, 36(2), $164-175$. 